\title{
Synthetic observations of internal gravity waves in the solar atmosphere
}

\author{
G. Vigeesh and M. Roth
}

\author{
Leibniz-Institut für Sonnenphysik (KIS), Schöneckstrasse 6, 79104 Freiburg, Germany \\ e-mail: vigeesh@leibniz-kis.de
}

Received 4 October 2019 / Accepted 9 December 2019

\begin{abstract}
Aims. We study the properties of internal gravity waves (IGWs) detected in synthetic observations that are obtained from realistic numerical simulation of the solar atmosphere.

Methods. We used four different simulations of the solar magneto-convection performed using the $\mathrm{CO}^{5} \mathrm{BOLD}$ code. A magneticfield-free model and three magnetic models were simulated. The latter three models start with an initial vertical, homogeneous field of 10,50 , and $100 \mathrm{G}$ magnetic flux density, representing different regions of the quiet solar surface. We used the NICOLE code to compute synthetic spectral maps from all the simulated models for the two magnetically insensitive neutral iron lines Fe I $\lambda \lambda 5434 \AA$ and $5576 \AA$. We carried out Fourier analyses of the intensity and Doppler velocities to derive the power, phase, and coherence in the $k_{\mathrm{h}}-\omega$ diagnostic diagram to study the properties of internal gravity waves.

Results. We find the signatures of the internal gravity waves in the synthetic spectra to be consistent with observations of the real Sun. The effect of magnetic field on the wave spectra is not as clearly discernible in synthetic observations as in the case of numerical simulations. The phase differences obtained using the spectral lines are significantly different from the phase differences in the simulation. The phase coherency between two atmospheric layers in the gravity wave regime is height dependent and is seen to decrease with the travel distance between the observed layers. In the studied models, the lower atmosphere shows a phase coherency above the significance level for a height separation of $\sim 400 \mathrm{~km}$, while in the chromospheric layers it reduces to $\sim 100-200 \mathrm{~km}$ depending on the average magnetic flux density.

Conclusion. We conclude that the energy flux of IGWs determined from the phase difference analysis may be overestimated by an order of magnitude. Spectral lines that are weak and less temperature sensitive may be better suited to detecting internal waves and accurately determining their energy flux in the solar atmosphere.
\end{abstract}

Key words. magnetohydrodynamics (MHD) - Sun: atmosphere - Sun: chromosphere - Sun: photosphere - Sun: magnetic fields waves

\section{Introduction}

Several observations have provided strong evidence for the presence of internal gravity waves (IGWs) in the solar atmosphere (Straus et al. 2008; Kneer \& Bello González 2011; Nagashima et al. 2014). These waves owe their existence to the proximity of an unstable layer adjacent to a stable environment. Penetrative flows from the unstable convective layer overshooting into the stable photosphere that lies above excite IGWs, among other magneto-atmospheric waves, and are thought to propagate higher up in the atmosphere.

In the low solar chromosphere, especially in the internetwork region, IGWs are thought to contribute to the ultraviolet background at spatial scales corresponding to meso-granulation (Straus \& Bonaccini 1997; Rutten \& Krijger 2003). Straus et al. (2008) reported that internal waves are found to be locally suppressed in strong magnetic field regions, while being abundantly present in magnetically quiet regions of the solar atmosphere. Despite providing a significant fraction of wave energy flux in the photosphere, the existence and role of IGWs in the upper layers of the atmosphere in the presence of magnetic field is still unclear. Newington \& Cally $(2010,2011)$ showed that the waves couple to other magneto-atmospheric waves.

More recent work by Vigeesh et al. (2017; 2019, hereafter Papers I and II) looked at IGWs generated in the solar atmosphere and investigated the effect of magnetic fields on their propagation. These latter authors showed that the emergent IGW spectra in the near-surface photospheric layer is unaffected by the presence or strength of the magnetic field. Internal gravity waves are generated with a considerable amount of wave flux independent of the average value of the magnetic flux density in the nearsurface region of the quiet Sun, where the waves likely originate. However, consistent with the observations, the propagation in the upper layers is influenced by the magnetic fields present there. It was shown that the subsequent coupling to Alfvénic waves is unlikely in a magnetic environment permeated with predominantly vertical fields, like in the network regions (see Borrero et al. 2017, for a recent review), and therefore they may not directly or indirectly contribute to the heating of low plasma$\beta$ layers (where the ratio of gas to magnetic pressure is less than unity) in the network-like regions of the solar atmosphere.

As their propagation properties are dependent on the average magnetic field in the higher layers, IGWs could be used to measure the average magnetic field properties of the upper solar atmosphere. Consequently, they may be used for monitoring the long-term evolution of the average magnetic field of the quiet Sun independently of direct magnetic field measurements. In this paper, we look more closely into the details of how these waves can be observed and discuss the implications of using them for diagnostic purposes. We use realistic numerical simulations of 
the solar atmosphere to compute the emerging spectra in the two iron lines Fe I $\lambda \lambda 5434 \AA$ and $5576 \AA$. Furthermore, we investigate the acoustic-gravity wave spectrum and compare it directly with real Sun observations.

The paper is organised as follows. In Sect. 2, we discuss the numerical models that we use. In Sect. 3, we describe the synthetic observables that are derived from the numerical models. A short description of the analysis is presented in Sect. 4. In Sect. 5, we report the results and discussion. The conclusion of the work is provided in Sect. 6 .

\section{Numerical simulations}

We carry out full forward modelling of near-surface solar magneto-convection using the $\mathrm{CO}^{5} \mathrm{BOLD}^{1}$ code (Freytag et al. 2012). The code solves the time-dependent non-linear magnetohydrodynamics (MHD) equations in a Cartesian box with an external gravity field, taking non-grey radiative transfer into account.

We study four models representing different regions of the solar surface convection. In each of the models, the computational domain is $38.4 \times 38.4 \times 2.8 \mathrm{Mm}^{3}$ in the $x \times y \times z$ direction, discretised on $480 \times 480 \times 120$ grid cells. The cell size in the horizontal is $80 \mathrm{~km}$ and the vertical cell size varies from $50 \mathrm{~km}$ in the lower part of the computational domain (convection zone) down to $20 \mathrm{~km}$ near the surface and in the atmosphere. The domain extends $\sim 1.3 \mathrm{Mm}$ above the mean Rosseland optical depth $\tau_{\mathrm{R}}=1$, providing the atmosphere wherein the waves propagate. The non-magnetic run (Sun-v0) is computed by setting the initial magnetic flux density to zero. The three magnetic runs, namely Sun-v10, Sun-v50, and Sun-v100, are computed by embedding the initial model with a uniform vertical field of 10,50 , and $100 \mathrm{G}$, respectively, in the entire domain. A detailed summary of the numerical models used in this work is described in Paper II.

Periodic boundary conditions are used for the side boundaries in all four models. The velocity field, radiation, and the magnetic field components are periodic in the lateral directions. The top boundary is open for fluid flow and outward radiation, with the density decreasing exponentially in the boundary cells outside the computational domain. The vertical component of the magnetic field is constant across the upper boundary and the transverse component drops to zero at the boundary. The bottom boundary is setup in such a way that the in-flowing material carries a constant specific entropy resulting in a radiative flux corresponding to an effective temperature ( $\left.T_{\text {eff }}\right)$ of $\sim 5770 \mathrm{~K}$. The bottom boundary conditions for the magnetic fields are the same as for the top boundary.

An equation of state that adequately describes the solar plasma, including partial ionisation effects, is used. The radiative transfer proceeds via an opacity binning method adapted from the MARCS stellar atmosphere package (Gustafsson et al. 2008), with the help of five opacity groups. For the radiative transfer we use long characteristics along a set of eight rays.

\section{Synthetic spectra}

We use the publicly available open-source, radiative transfer code NICOLE ${ }^{2}$ (Socas-Navarro et al. 2015) to compute synthetic spectra in local thermodynamic equilibrium (LTE). The code solves the equation of radiative transfer along onedimensional (1D) vertical columns assuming a plane-parallel

1 CO5BOLD stands for "COnservative COde for the COmputation of COmpressible COnvection in a BOx of L Dimensions with $\mathrm{L}=2$, 3." In this work we use $\mathrm{L}=3$.

2 https://github.com/hsocasnavarro/NICOLE
Table 1. Atomic parameters used for the synthesis of the spectral maps

\begin{tabular}{lcc}
\hline \hline & Fe I $\lambda 5434 \AA$ & Fe I $\lambda 5576 \AA$ \\
\hline Central wavelength $(\AA)$ & 5434.5235 & 5576.0884 \\
Excitation potential $(\mathrm{eV})$ & 1.0110557 & 3.43019 \\
$\log (g f)$ & -2.122 & -0.94 \\
Transition & ${ }^{5} \mathrm{~F}_{1}-{ }^{5} \mathrm{D}_{0}$ & ${ }^{5} \mathrm{~F}_{1}-{ }^{5} \mathrm{D}_{0}$ \\
Collisions & Barklem & Barklem \\
Damping sigma & 243 & 854 \\
Damping alpha & 0.247 & 0.232 \\
\hline
\end{tabular}

Notes. The spectroscopic data of the selected lines are taken from the National Institute of Standards and Technology (NIST) Atomic Spectra Database (Kramida et al. 2018) and the data for collisional broadening by hydrogen are from Barklem et al. (1998).

atmosphere. NICOLE is capable of non-LTE (NLTE) radiative transfer calculations, but we are mainly interested in the properties of the waves propagating in the low photosphere where the assumption of LTE is still valid. However, a proper treatment of the upper layers will require NLTE analysis, which we defer to a future study.

The 3D snapshots from the numerical simulation are interpolated to a constant $z$-grid with $\Delta z=10 \mathrm{~km}$. The density, gas pressure, and velocity on the corresponding Rosseland mean optical depth grid are then passed as the model atmosphere to the synthesis code. The code uses its native equation of state (EOS) to calculate the electron pressure. We use the same set of abundances that are used in the $\mathrm{CO}^{5} \mathrm{BOLD}$ simulation. The radiative transfer is then carried out for each 1D column $(480 \times 480)$ in the horizontal direction independently and for each snapshot. The spectral lines are sampled at $5 \mathrm{~m} \AA$ resolution spanning a wavelength range of $\pm 500 \mathrm{~m} \AA$ around the central wavelength.

\subsection{Line selection}

We consider two magnetically insensitive neutral iron lines Fe I $\lambda \lambda 5434 \AA$ and $5576 \AA$, both of which have a Landé $g$ factor equal to zero. The atomic parameters of our selected lines are listed in Table 1. Figure 1 shows the average profile of the selected lines from a snapshot of the non-magnetic (Sun-v0) and one of the magnetic models (Sun-v100) along with the Fourier transform spectrometer (FTS) atlas spectra for the lines. The $95 \%$ bounds of the spectral profile over the whole domain in the selected snapshot are also shown. Our choice of the spectral lines is based on the work by Kneer \& Bello González (2011), where these two Fe I lines were used to estimate the energy transport by acoustic and atmospheric gravity waves in the quiet Sun.

The two lines differ mainly in their temperature sensitivity; the low-excitation potential Fe I $\lambda 5434 \AA$ line is more temperature sensitive than the Fe I $\lambda 5576 \AA$ line, the latter being otherwise considered to be temperature insensitive (Cabrera Solana et al. 2005). The line-forming surface of the Fe I $\lambda 5434 \AA$ line is therefore less smooth than that of the Fe I $\lambda 5576 \AA$ line; this has a more adverse effect on the phase difference analysis that is used to investigate waves, a point to which we come later in Sect. 5.

\subsection{Synthetic Dopplergrams and velocity response function}

To study IGWs, we primarily examine the $2 \mathrm{D}$ velocity field in the solar atmosphere, which can be readily obtained from the Doppler shift of observed spectral lines. The major drawback is 
(a)

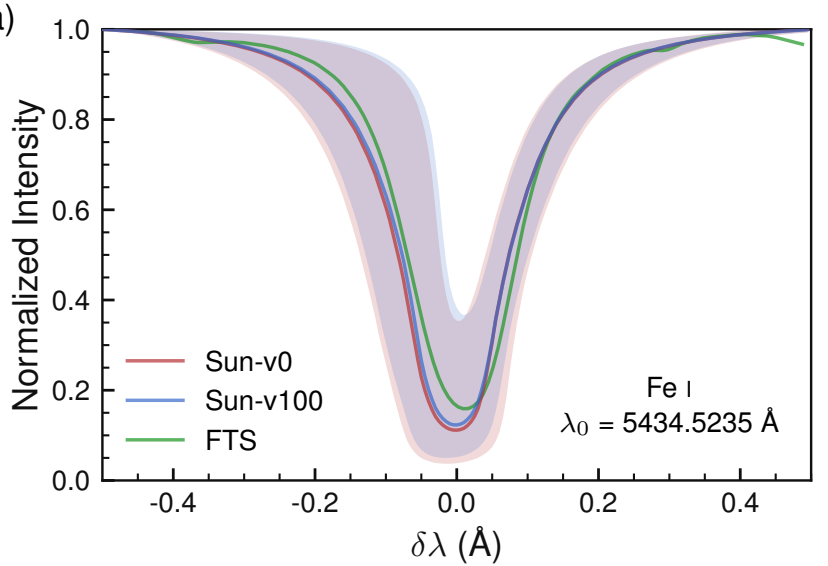

(b)

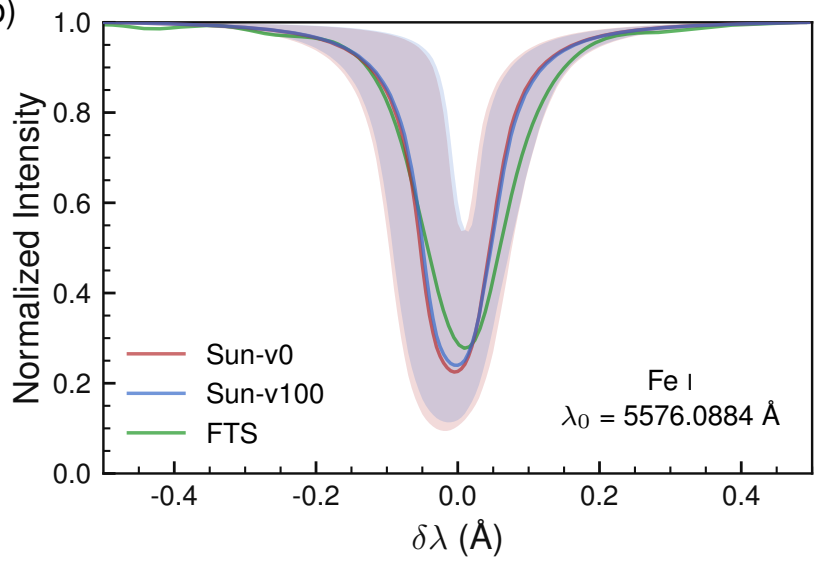

Fig. 1. Median profile of (a) Fe I $\lambda 5434 \AA$ and $(b)$ Fe I $\lambda 5576 \AA$ taken from a snapshot of the Sun-v0 (blue) and Sun-v100 (red) models. The FTS atlas spectra for the respective lines are shown in green. The background shaded region shows the $95 \%$ bounds of the line profiles over the whole computational domain in the selected snapshot.

that they provide an average of the velocity field over a broad height range, effectively producing a smeared out form of the velocity perturbations caused by any passing wave. Nevertheless, the line shifts can be estimated using different techniques from the same line profile which provide velocity measurements corresponding to different atmospheric layers. In this work, we estimate three different Doppler velocities from the synthesised spectral lines. Our first approach is to use the Fourier method, where the phase of the first Fourier component of the line profile gives us the Doppler shift. In the second approach, we estimate the velocity from the shift in the centre of gravity (COG) of the line. Since these two approaches look at the overall shift in the spectral line, they give similar values in terms of where the velocity signal is formed. In this paper, we mainly discuss results from the COG method. Lastly, we compute the shift of the line profile minima by fitting a second-degree polynomial in the range $\pm 30 \mathrm{~m} \AA$ around the minimum position of the spectral line. The line minima method gives us the velocity field in the higher layers when compared to the Fourier or the COG method. Some of the aforementioned methods have been discussed in the literature (Scherrer et al. 1995; Fleck et al. 2011; Couvidat et al. 2012; Nagashima et al. 2014).

We now look at the velocity response function $\left(R F_{\mathrm{v}}\right)$ to estimate the height of formation of the velocity signals obtained from the different methods described above. The response functions are calculated using the method described (a)

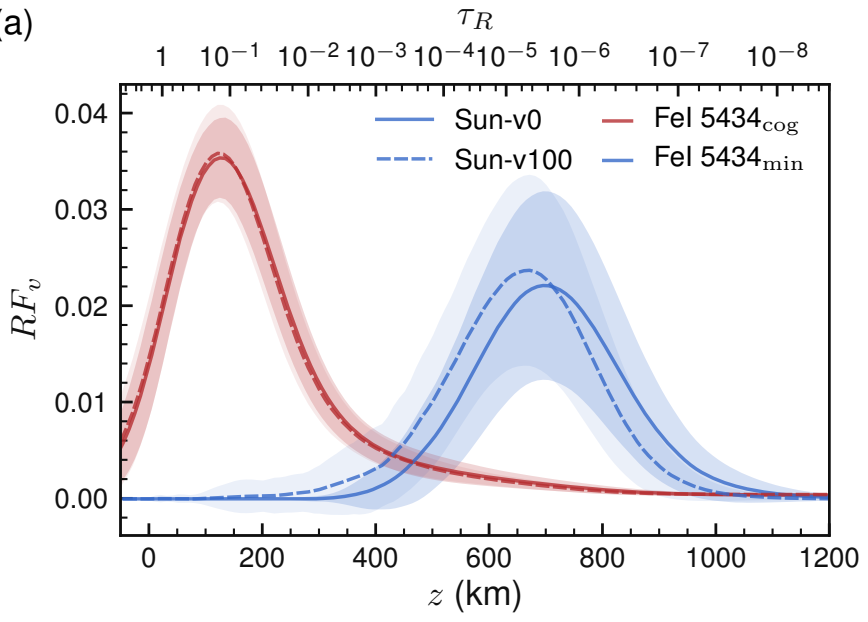

(b)

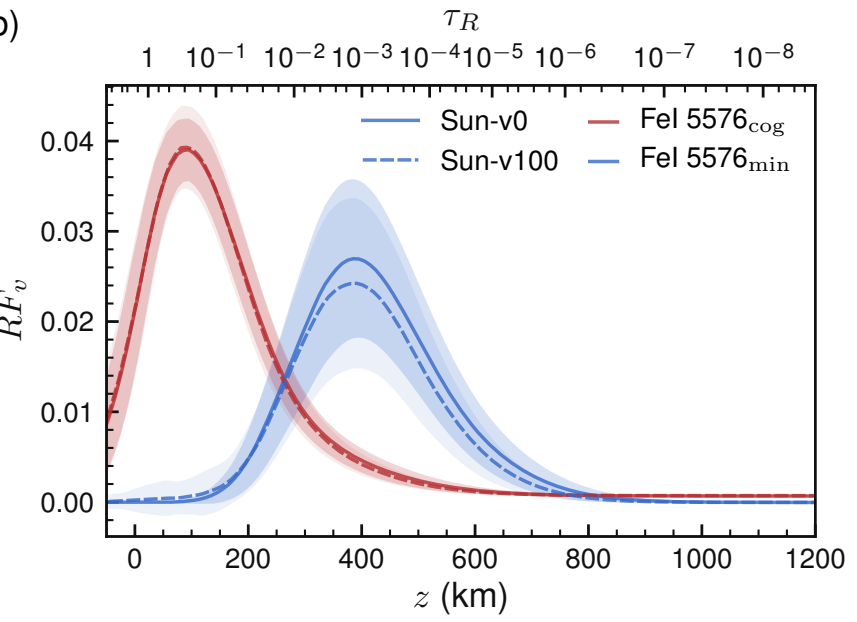

Fig. 2. Mean $R F_{\mathrm{v}}$ of the velocities determined using the COG shift (red) and the line-core shift (blue) for the Fe I $\lambda 5434 \AA$ (a) and Fe I $\lambda 5576 \AA$ (b) lines in the non-magnetic model (Sun-v0; solid) and in a magnetic model (Sun-v100; dashed). The spread of the $R F_{\mathrm{v}}$ ranging from an intergranular to granular region is represented by the background shade with the fainter shade representing the magnetic model.

in Eibe et al. (2001). In Fig. 2a, we compare the mean $R F_{\mathrm{v}}$ for the Fe I $\lambda 5434 \AA$ line in the non-magnetic model (Sun-v0) and in one of the magnetic models (Sun-v100), both obtained using the COG method and the line minima method. The spread of the $R F_{\mathrm{v}}$ ranging from the inter-granular to the granular region is represented by the shaded background with the fainter shade representing the magnetic model. The corresponding profiles for the Fe I $\lambda 5576 \AA$ are shown in Fig. $2 b$. We see that the velocity obtained using the COG method is formed mainly close to the surface around $z=100$. There is a small difference in the height of formation for the two lines of $\sim 30 \mathrm{~km}$, with the Fe I $\lambda 5434 \AA$ line forming slightly higher up in the atmosphere. We do not see any difference in formation height between the non-magnetic and the magnetic models. The line-minima method on the other hand probes the higher layers, as evident from the corresponding $R F_{\mathrm{v}}$ (blue curves) shown in Fig. 2. The peak of the $R F_{\mathrm{v}}$ for the two Fe I lines is separated by $280-310 \mathrm{~km}$, with the magnetic models peaking lower than their non-magnetic counterpart in the case of Fe I $\lambda 5434 \AA$. The $R F_{\mathrm{V}}$ for the Fe I $\lambda 5576 \AA$ line using the COG method that we computed using our simulation models matches that of Berrilli et al. (2002). The $R F_{\mathrm{v}}$ for the line-minima in our case is formed $50 \mathrm{~km}$ above compared to Berrilli et al. (2002) and approximately at the same height as in 


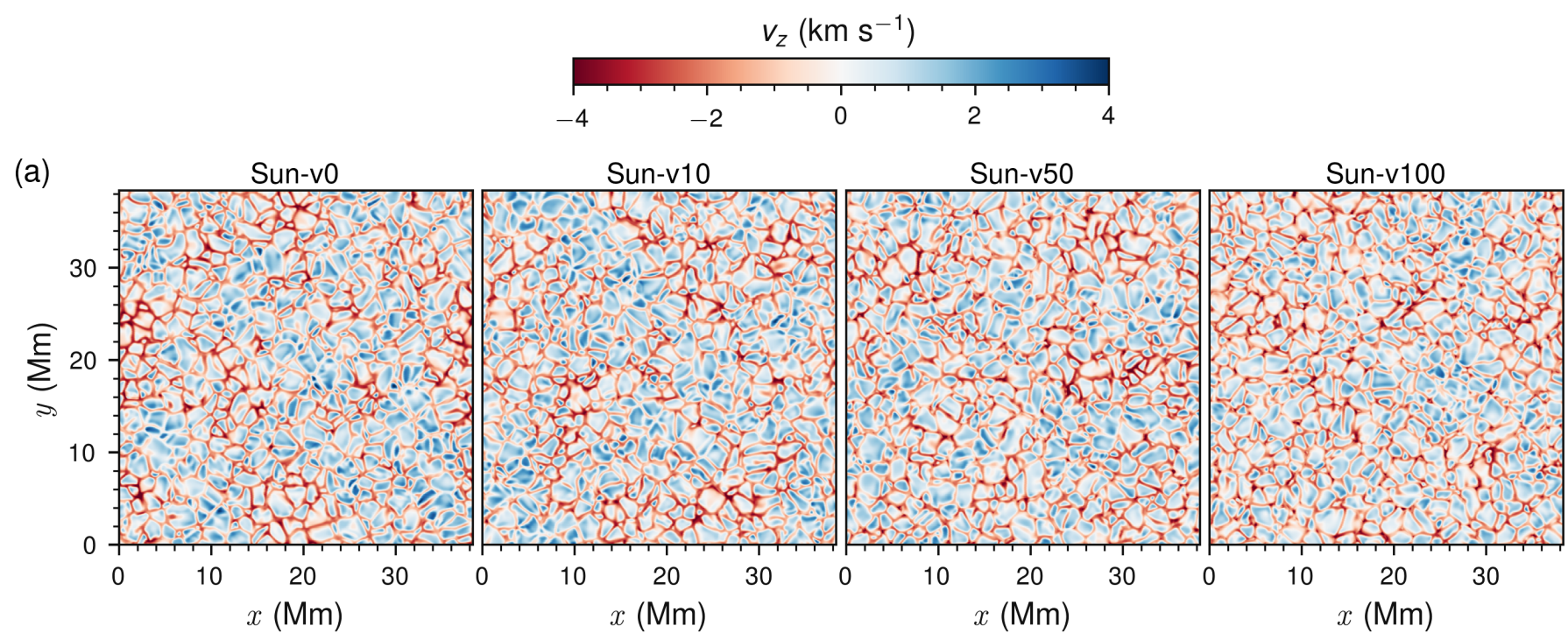

(b)
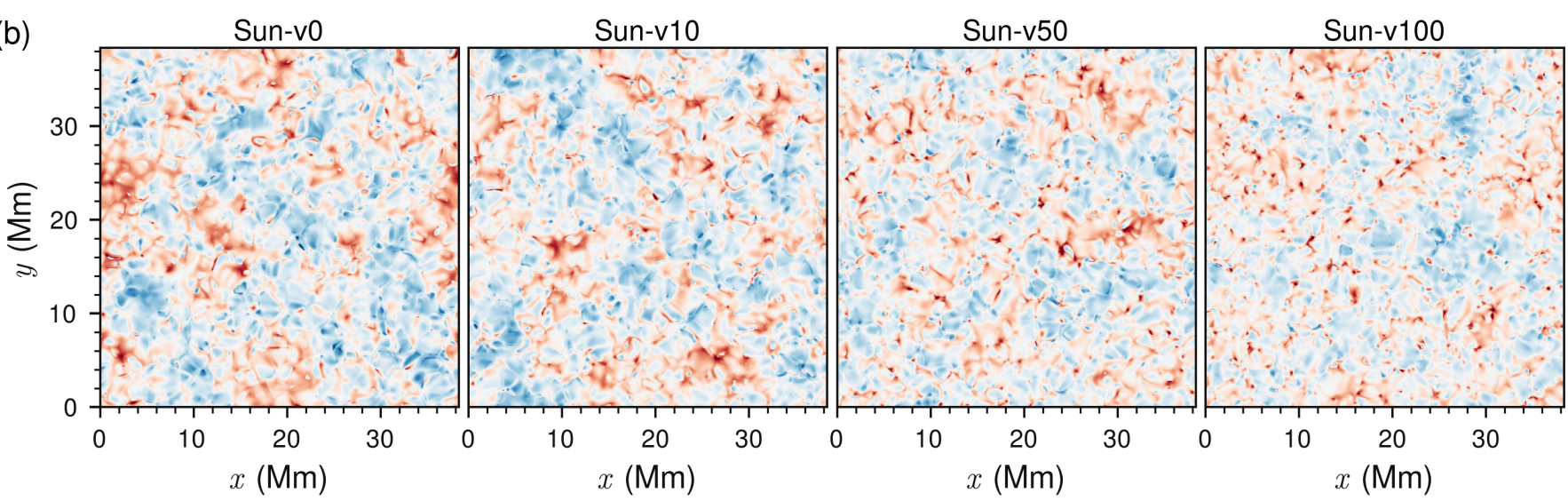

Fig. 3. Snapshot of Dopplergrams from Fe I $\lambda 5576 \AA$ for the four different models (left to right: increasing average magnetic flux density from 0 to $100 \mathrm{G}$ ), estimated using $(a)$ the COG method which provides the velocity field around $z=100 \mathrm{~km}$ and $(b)$ the line-minima shift which provides the velocity field around $z=400 \mathrm{~km}$

the case of Bello González et al. (2009). We also compared the $R F_{\mathrm{v}}$ for the Fe I $\lambda 5434 \AA$ A line with that of Bello González et al. (2010) and Kneer \& Bello González (2011) and see that the lineforming peaks are systematically shifted higher up in our case by around $100 \mathrm{~km}$. The discrepancies may be due to the absence of a chromospheric temperature rise in our models or due to the assumption of LTE. A representative snapshot of the Dopplergrams for the $\mathrm{Fe}$ I $\lambda 5576 \AA$ Aine obtained using the COG method and the line-minima method is shown in Fig. 3 showing the difference in the velocity field as a result of probing different layers using the same spectral line.

\section{Analysis}

Now that we have computed the different Doppler velocities and estimated the height of formation of the velocity signals for each line, we can now look for signatures of waves in the velocity field. We carry out a spectral analysis of the 2D synthetic observations by Fourier-transforming the physical quantities in both space and time to identify and separate IGWs from other types of waves present in the domain. The analysis methodology is already presented in Paper I and Paper II. In the foregoing work, we investigated the wave signatures from the velocity signals obtained directly from the simulation box. The analyses was carried out at constant geometrical height, thereby allowing us to study the wave generation and propagation as it happens in the physical volume. In this work, we are nevertheless interested in the effect of probing layers of constant optical depth scale (through information provided by spectral lines) on the inferred properties of the waves. The levels of constant optical depth are highly corrugated in the atmosphere and therefore it is still unclear what effect they may have on the observations of waves. Therefore, it is important to study the behaviour of velocities at constant (Rosseland) optical depth interpolated directly from the simulations and to see if one is able to still recover the signature of IGW propagation and the effect of magnetic field. This will enable us to properly interpret the spectral signatures obtained from the estimates of velocities derived from the spectral lines. We investigate the velocity-velocity $(v-v)$ phase difference and the related coherence spectra obtained from different velocity measurements with the aim of comparing them with observations.

In this work, we use three-hour time-series of the synthetic maps and the domain spans $38.4 \mathrm{Mm}$ in the horizontal direction. This gives us a frequency resolution $(\Delta v)$ of $92.6 \mu \mathrm{Hz}$ and a wavenumber resolution $\left(\Delta k_{\mathrm{h}}\right)$ of $0.164 \mathrm{rad} \mathrm{Mm}{ }^{-1}$. The grid resolution of $80 \mathrm{~km}$ results in a Nyquist wavenumber $\left(k_{\mathrm{Ny}}=\pi / \delta x\right)$ of $39.25 \mathrm{rad} \mathrm{Mm}^{-1}$ of which we are only interested in horizontal wavenumbers up to $10 \mathrm{rad} \mathrm{Mm}^{-1}$, where the bulk of IGWs occur. A vertical and horizontal grid constant of, respectively 20 


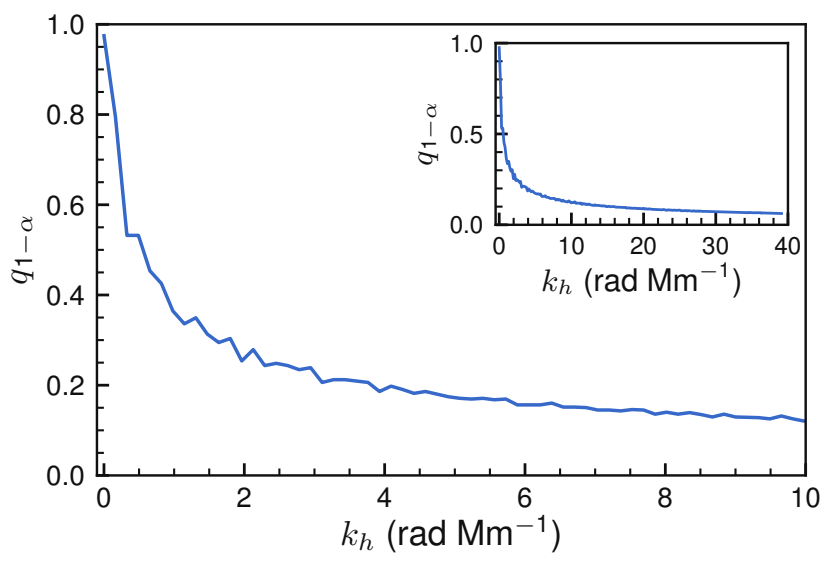

Fig. 4. Zero-coherence threshold as a function of $k_{\mathrm{h}}$ at the significance level $\alpha=0.05$. The inset plot shows the entire range up to the Nyquist wavenumber $\left(k_{\mathrm{Ny}}=39.25 \mathrm{Mm}^{-1}\right)$.

and $80 \mathrm{~km}$ is sufficient to capture the range of the internal wave spectrum in the models as discussed in Paper I. Snapshots from the simulations are taken at $30 \mathrm{~s}$ intervals, and the spectral line synthesis is performed for each snapshot, resulting in a Nyquist frequency $\left(v_{\mathrm{Ny}}\right)$ of $16.66 \mathrm{mHz}$.

\subsection{Zero coherence threshold}

Coherence measures the linear dependence of the two processes, which in our case is the velocity signals at two different heights. However, even for two independent processes, the coherence estimator will give a non-zero value due to fact that the spectra are computed by averaging of several individual segments. We consider a confidence threshold with a certain probability such that if the estimated coherence is below this threshold, zero coherence is indicated for that Fourier component. Following Hannan (1990), the zero coherence threshold $\left(q_{1-\alpha}\right)$ at the significance level $\alpha$ can be expressed as

$q_{1-\alpha}=\sqrt{1-\alpha^{2 /\left(N_{\mathrm{s}}-2\right)}}$,

where $N_{\mathrm{s}}$ is the equivalent number of degrees of freedom in the case of independent segments. If the estimated coherence $\mathcal{K}>$ $q_{1-\alpha}$, the null hypothesis of zero coherency can be rejected with a $100 \alpha \%$ probability of error. In our case, $N_{\mathrm{s}}$ can be taken as the number of averages performed for a given $k_{\mathrm{h}}$ in the $k_{x}-k_{y}$ plane. Figure 4 shows the zero coherence threshold as a function of $k_{\mathrm{h}}$ at the significance level $\alpha=0.05$. The threshold decreases with $k_{\mathrm{h}}$ due to the increasing number of averages in the $k_{x}-k_{y}$ plane and falls below 0.5 for $k_{\mathrm{h}}>1 \mathrm{rad} \mathrm{Mm}^{-1}$. The uneven curve is the result of rasterisation in the $k_{x}-k_{y}$ plane.

\subsection{Confidence interval for the phase and coherence}

In this study, we also consider confidence intervals for the measured phase and coherence. The 100(1- $\alpha) \%$ confidence interval for the estimated phase $(\hat{\phi})$ is given by the inequality (Hannan 1990)

$|\sin (\hat{\phi}-\phi)| \leq\left\{\frac{1-\mathcal{K}^{2}}{\mathcal{K}^{2}\left(2 N_{\mathrm{s}}-2\right)}\right\}^{1 / 2} t_{2 N_{\mathrm{s}}-2}\left(\frac{\alpha}{2}\right)$,

where $t_{2 N_{\mathrm{s}}-2}\left(\frac{\alpha}{2}\right)$ is the $(1-\alpha / 2)^{\mathrm{th}}$ quantile of the $t$-distribution with $2 N_{\mathrm{s}}-2$ degrees of freedom.
An approximation for the 100(1- $\alpha) \%$ confidence interval for the estimated coherence $(\mathcal{K})$ is given as (Hannan 1990)

$q_{l, u}=\tanh \left\{\tanh ^{-1}(\mathcal{K}) \pm\left(u_{\alpha / 2}\right)\left(2\left(N_{s}-1\right)\right)^{-1 / 2}-\left(2\left(N_{\mathrm{s}}-1\right)\right)^{-1}\right\}$

where $u_{\alpha / 2}$ is the $(1-\alpha / 2)^{\text {th }}$ quantile of the standard normal distribution.

\subsection{Energy flux estimation}

The energy flux of the IGWs can be directly determined from the simulation as described in Paper I and Paper II. However, in this study we are interested in the diagnostic information provided by the observed spectral lines and in determining the energy flux using the phase difference of the Doppler velocity measurements.

Given the difference between the formation heights $(\Delta z)$ of the two lines, the vertical phase velocity spectrum is determined from the phase difference spectra as

$v_{\mathrm{ph}, \mathrm{z}}=\Delta z / \Delta t$,

where $\Delta t=\Delta \Phi / \omega$ represents the vertical phase travel time between the two layers provided that the medium through which the wave propagates is homogeneous. Here, $\Delta \Phi$ represents the phase difference in radians between the two layers for a harmonic wave of frequency $\omega$. The vertical group velocity spectrum is then determined from the phase velocity as (Straus et al. 2008; Kneer \& Bello González 2011)

$v_{\mathrm{g}, \mathrm{z}}=-v_{\mathrm{ph}, \mathrm{z}} \sin ^{2} \theta$,

where $\theta$ is the angle between the horizontal and the wavevector $\boldsymbol{k}$, given using the dispersion relation as $\cos \theta=\omega / N$ in the incompressible limit, with $N$ representing the Brunt-Väisälä frequency. Finally, we obtain the vertical energy flux spectrum as the product of energy density and vertical group velocity,

$\mathcal{F}_{\mathrm{z}}=\frac{1}{2} \varrho\left\langle v^{2}\right\rangle v_{\mathrm{g}, \mathrm{z}}$

\section{Results and discussion}

We now present results from our spectral analysis. First, we analyse the intensity maps and compute the power, phase, and coherence on the $k_{\mathrm{h}}-\omega$ diagnostic diagram. We then turn to the analysis of the velocity data, presenting first the results from constant optical depth and then those of the spectral analysis of the synthetic Dopplergrams. We compare the energy flux estimation from the constant optical depth measurements and the Dopplergrams.

\subsection{Power, phase difference, and coherence spectra of intensity}

As an illustration of the importance of multi-spectral observations for the study of IGWs, we first look at the diagnostic spectra of intensity obtained from the synthetic observations of the non-magnetic model (Sun-v0). In Fig. 5, the leftmost plot shows the $k_{\mathrm{h}}-\omega$ power spectra of the line core intensity of the Fe I $\lambda 5434 \AA$ line. The second and third plots show the phase and coherence spectra, respectively, between the line core intensities of Fe I $\lambda 5576 \AA$ and Fe I $\lambda 5434 \AA$. The rightmost plot shows the power as a function of $k_{\mathrm{h}}$ integrated over the IGW region for the continuum intensity and line core intensities of 
(a) Power

(Fe I 5434, line core)

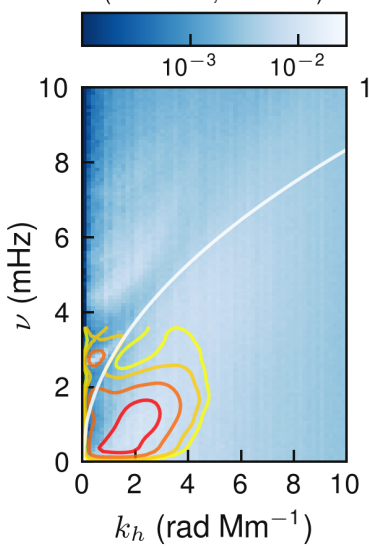

(b) Phase difference
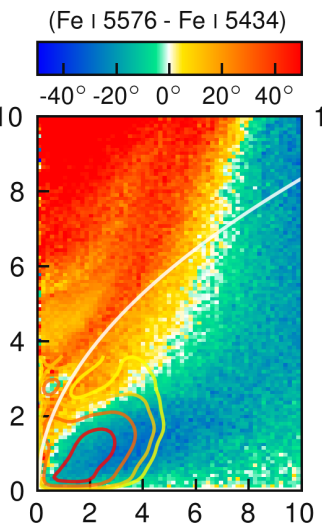

$k_{h}\left(\mathrm{rad} \mathrm{Mm}^{-1}\right)$ (c) Coherence

(Fe । 5576 - Fe I 5434)

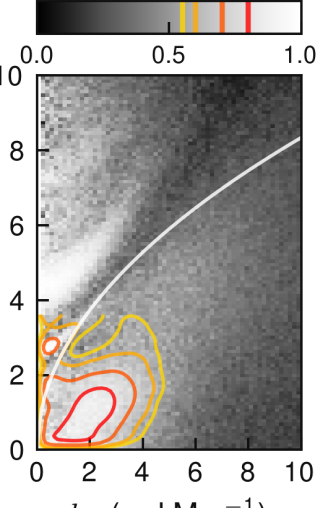

(d)

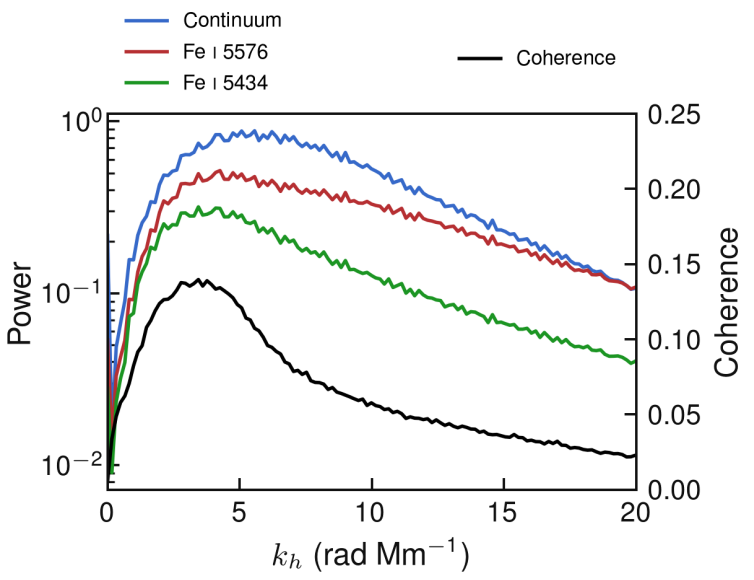

Fig. 5. (a) Power in the $k_{\mathrm{h}}-\omega$ diagnostic diagram of the line core intensity of the Fe I $\lambda 5434 \AA$ line. The phase $(b)$ and coherence $(c)$ spectra between the line-core intensity of the Fe I $\lambda 5576 \AA$ and Fe I $\lambda 5434 \AA$ lines are also shown. The white line marks the dispersion relation of the surface gravity waves $(f$-mode). The coloured contour lines in $(a)-(c)$ mark the coherence levels with the reference colours shown in the colourmap of $(c)$. (d) Power as a function of $k_{\mathrm{h}}$ integrated over the IGW region for the continuum intensity and line-core intensities of the Fe I $\lambda \lambda 5576 \AA$ and $5434 \AA$ lines. Also shown in black is the mean coherence in the IGW region.

Fe I $\lambda \lambda 5576 \AA$ and $5434 \AA$ lines. The mean coherence over the IGW region as a function of $k_{\mathrm{h}}$ is shown in black. It is clear from the power spectra that, whether it be the continuum intensity which is formed low in the atmosphere, or the line core which is formed in the higher layers, the power in the IGW region is dominated by convective velocity signal from the super granular scales $\left(k_{\mathrm{h}} \lesssim 1 \mathrm{rad} \mathrm{Mm}^{-1}\right)$ to the granular scales $\left(k_{\mathrm{h}} \gtrsim 2 \mathrm{rad} \mathrm{Mm}^{-1}\right)$. The signature of the coherent IGW waves $\left(k_{\mathrm{h}} \approx 1-4 \mathrm{rad} \mathrm{Mm}^{-1}\right)$ with their characteristic downward phases (see, for e.g. Paper I) cannot be discerned in the power spectra. This is evident from the location of the enhanced coherence in Fig. 5 d relative to the power spectra. To make this point clear, we over-plotted contour lines of four coherency values greater than 0.5 on the power spectra and the phase spectra in Figs. $5 \mathrm{a}-\mathrm{c}$. An internal wave transporting energy at an angle to the vertical, with an upward component, will have a downward-propagating phase component that shows up as a negative phase lag between two geometrical heights. The negative phases (blue-green) within the contour clearly show that this region marks the presence of upward propagating IGWs, which are otherwise indistinguishable in the power spectra. This highlights the importance of simultaneous or quasi-simultaneous 2D narrowband imaging by scanning over a single line or multiple lines in order to study IGWs in the solar atmosphere. Our focus in the subsequent sections is on the analysis of the velocity measurement obtained from the two Fe I lines.

\subsection{Phase and coherence spectra at constant optical depth}

We start with the velocity measurements interpolated from the simulation domain on to surfaces of constant mean (Rosseland) optical depth. In Fig. 6, we show the phase and coherence spectra between two pairs of velocity measurements each taken at a constant optical depth layer for all the four models studied here. We select three representative layers, namely $\tau_{\mathrm{R}}=0.5,1.0 \times 10^{-3}, 5.0 \times 10^{-6}$. These correspond to the layers close to where the different velocity response functions for the two lines show a peak as shown in Fig. 2 in Sect. 3.

We first look at the phase and coherence spectra for the pair $\tau_{\mathrm{R}}=0.5$ and $1.0 \times 10^{-3}$ in Fig. $6 \mathrm{a}$. The blue curves mark the propagation boundary of acoustic-gravity waves for the top (solid) and bottom (dashed) layers, with the IGWs located below the lower propagation boundary. Here we use the isothermal cut-off frequencies (see Paper I). Although all the four models show a downward phase propagation in the IGW regime, an apparent difference in the phases due to the effect of magnetic field is evident when we compare the four different models. The decrease in the phase difference in the IGW regime is probably due to the decreasing geometrical height difference between the two constant $\tau_{\mathrm{R}}$ layers with the increasing average magnetic field. We also notice that beyond $k_{\mathrm{h}}=8 \mathrm{rad} \mathrm{Mm}^{-1}$ the phase spectra become more noisy in all the models, which provides hints about the spatial scales over which the optical depth surface may show drastic fluctuations. However, we see from the coherence spectra for this pair of layers that the coherence is close to 0 for $k_{\mathrm{h}}>5 \mathrm{rad} \mathrm{Mm} \mathrm{Mm}^{-1}$. Therefore, we may deduce that a reliable estimate of the phase spectra can only be taken for $k_{\mathrm{h}}$ up to $5 \mathrm{rad} \mathrm{Mm} \mathrm{Mm}^{-1}$. Tracing the dispersion relation curve of the surface gravity waves ( $f$-mode) shown in grey reveals an apparent phase difference in the non-magnetic model that is completely absent from stronger magnetic models.

Looking now at the phase and coherence spectra for the pair $\tau_{\mathrm{R}}=1.0 \times 10^{-3}$ and $5.0 \times 10^{-6}$ shown in Fig. $6 \mathrm{~b}$, we indeed see the signature of upward phase propagation in the strong magnetic model as a result of downward-propagating waves. However, this downward propagation is rather restricted to the region below $v=2 \mathrm{mHz}$. In the region above $v=2 \mathrm{mHz}$ (within the IGW regime) we notice that the phases are positive, but the coherence spectrum at these locations is below the zero coherence threshold, and therefore the phase measurements are unreliable. The surface gravity waves ( $f$-mode) show an almost zero phase difference in the non-magnetic model but are, like in the lower layers, completely absent in stronger magnetic models.

\subsection{Phase and coherence spectra from Doppler velocities}

Now we turn to the analysis of wave spectra from the Doppler velocities estimated from the synthetic spectral lines. First, we study the phase and coherence spectra between Doppler velocities from a single line employing the different definitions of the Doppler velocity described in Sect. 3.2. We compare the lineminima and COG method which gives us the velocity field at different layers in the atmosphere. This is followed up by looking 
G. Vigeesh and M. Roth: Synthetic observations of internal gravity waves in the solar atmosphere

(a)
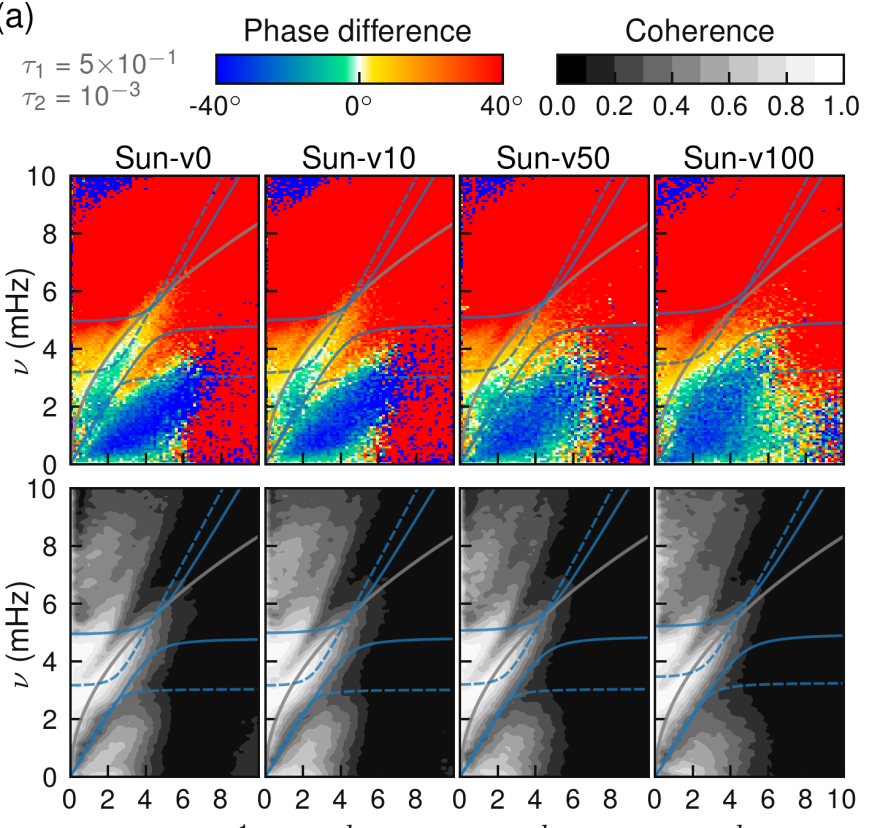

$k_{h}\left(\operatorname{rad~} \mathrm{Mm}^{-1}\right)$
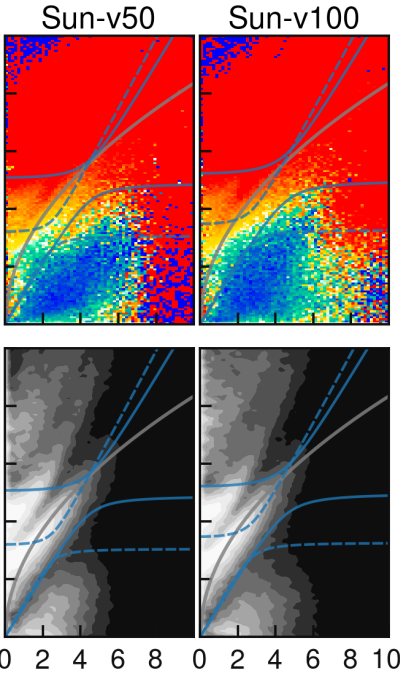

$k_{h}$ (b)
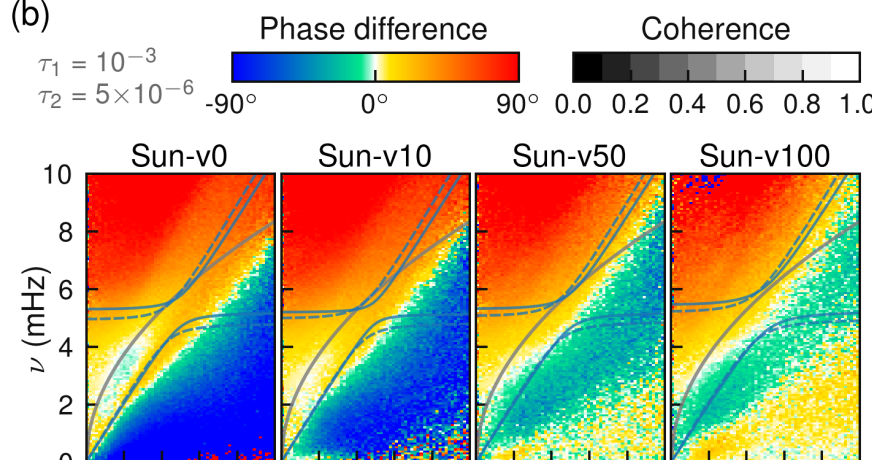

Sun-v50

Sun-v100
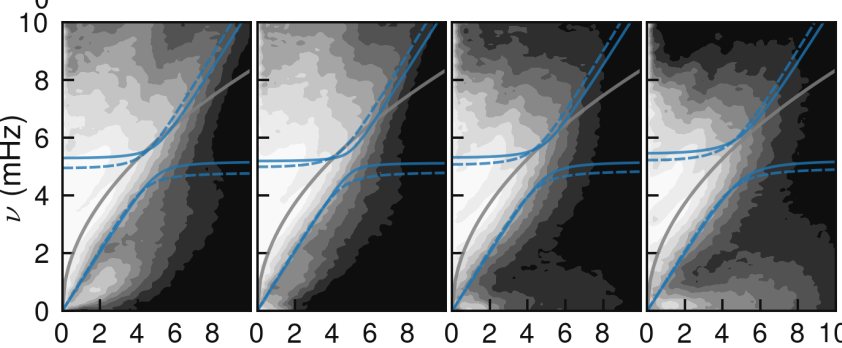

$k_{h}\left(\operatorname{rad~} \mathrm{Mm}^{-1}\right)$

$k_{h}$

$k_{h}$

$k_{h}$

Fig. 6. Phase difference (colour) and coherence (greyscale) spectra between layers of constant optical depth $(a) \tau_{\mathrm{R}}=0.5$ and $1.0 \times 10^{-3}$ and (b) $\tau_{\mathrm{R}}=1.0 \times 10^{-3}$ and $5.0 \times 10^{-6}$. The grey curve marks the dispersion relation of the surface gravity waves. The blue curves mark the propagation boundary of acoustic-gravity waves for the top (solid) and bottom (dashed) layers.

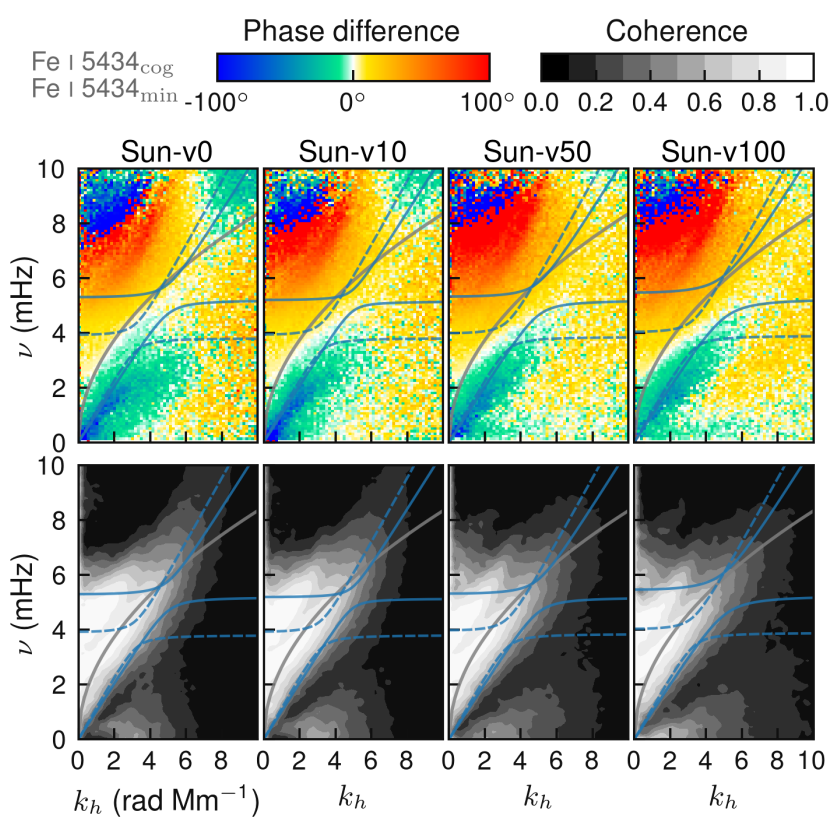

Fig. 7. Phase difference (colour) and coherence (greyscale) spectra for Fe I $\lambda 5434 \AA$ line measured with the velocities derived using the COG and line-minima shifts. The grey curve marks the dispersion relation of the surface gravity waves. The blue curves mark the propagation boundary of the acoustic-gravity wave corresponding to the approximate formation heights where the COG (dashed) and line-minima (solid) velocities are measured.

at the phase and coherence spectra of two separate lines utilising either the COG shift or the line-core Doppler shifts.

In Fig. 7, we show the phase and coherence spectra for the Fe I $\lambda 5434 \AA$ line. Here, the Doppler velocities obtained using the COG method and the line-minima method are used for computing the phase and coherence spectra. According to

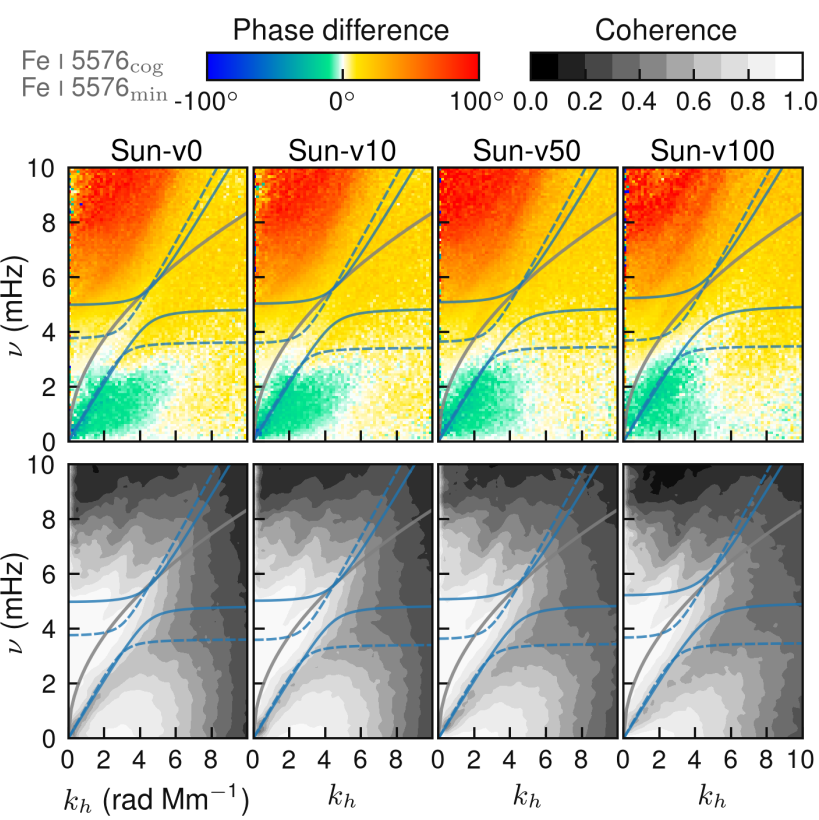

Fig. 8. Phase difference (colour) and coherence (greyscale) spectra for the $\mathrm{Fe}$ I $\lambda 5576 \AA$ line measured with the velocities derived using the COG and line-minima shifts. The grey curve marks the dispersion relation of the surface gravity waves. The blue curves mark the propagation boundary of the acoustic-gravity wave corresponding to the approximate formation heights where the COG (dashed) and line-minima (solid) velocities are measured.

the $R F_{\mathrm{v}}$ shown in Fig. 2a the two velocity signals are separated by around $550-570 \mathrm{~km}$. As a result of this large separation the coherency is very low, especially in the IGW region. The effect of magnetic field is negligible in the phase spectra, with the Sun-v100 model showing a marginal upward phase propagation (see area covered in yellow within the IGW regime 
(a)
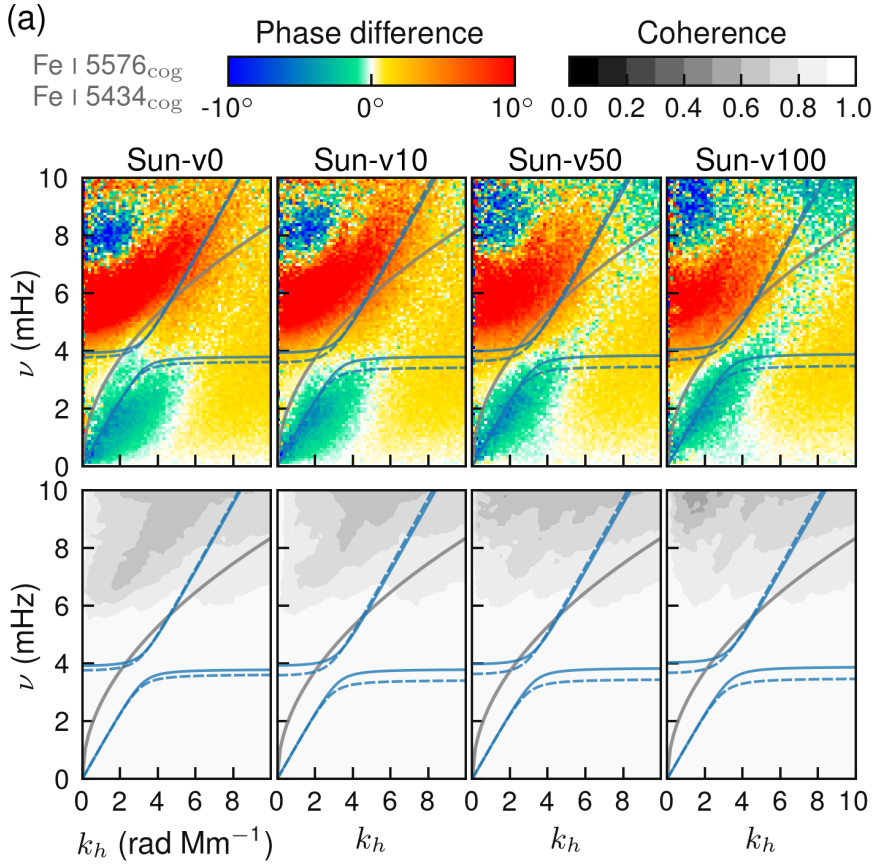

(b)
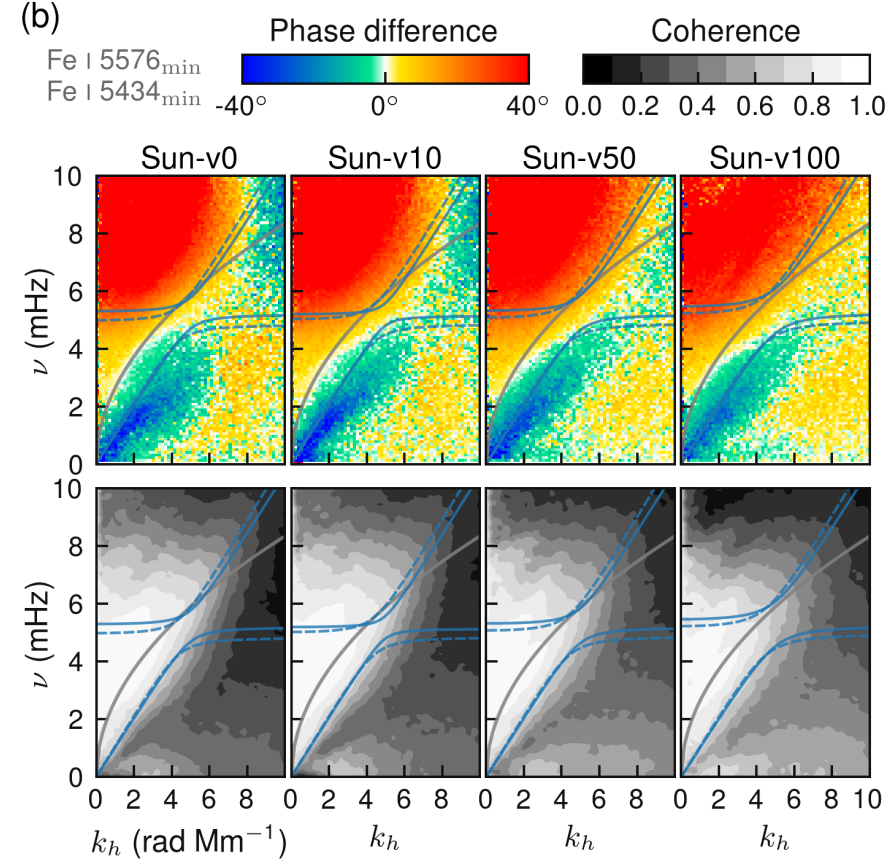

Fig. 9. Phase difference (colour) and coherence (greyscale) spectra estimated from the (a) velocities measured using the COG shift of Fe I $\lambda 5434 \AA$ and Fe I $\lambda 5576 \AA$ and $(b)$ velocities measured using the line-minima shift of Fe I $\lambda 5434 \AA$ and Fe $\mathrm{I}$ 5576 $\mathrm{A}$. The grey curve marks the dispersion relation of the surface gravity waves. The blue curves mark the propagation boundary of the acoustic-gravity wave corresponding to the approximate formation heights where the COG (dashed) and line-minima (solid) velocities are measured.

compared to the Sun-v0 model) for wave numbers larger than $k_{\mathrm{h}}=4 \mathrm{rad} \mathrm{Mm}^{-1}$. However, the low coherence in this region renders any phase estimate unreliable.

In Fig. 8, we show the phase and coherence spectra for the Fe I $\lambda 5576 \AA$ line. The separation between the peak of $R F_{\mathrm{v}}$ for the COG and line-minima is much less in this case (290-300 km) compared to that of the $\mathrm{Fe} I \lambda 5434 \AA$ line. As a consequence of this, the coherence is stronger for this line. Additionally, the fact that Fe I $\lambda 5576 \AA$ is less temperature sensitive adds to the stronger coherence between the layers, as the line-forming layers are less corrugated. The influence of magnetic field on the phase difference spectra is barely discernible except for the change in sign in the IGW region for wave numbers in the range of 4$6 \mathrm{rad} \mathrm{Mm}^{-1}$ for frequencies above $v=1 \mathrm{mHz}$ (clearly seen when comparing the yellow area in the IGW region in Sun-v100 to the green area in the Sun-v0 case).

We now turn to the phase and coherence spectra as obtained from looking at velocity signals from two separate spectral lines. In Fig. 9 we show the phase difference between the Fe I $\lambda 5576 \AA$ and Fe I $\lambda 5434 \AA$ lines using both the COG method (left) and line-minima (right), separately. We see that the coherency has increased as a result of probing surfaces separated by a shorter distance, for instance $30 \mathrm{~km}$ for the COG method and $280-310 \mathrm{~km}$ for the line-minima method. This shows that the phase spectra are more reliable when two different lines are considered that are separated by a rather close formation height, provided the phase difference is still measurable. We see that for the line-minima, the phase spectra show a hint of the effect of magnetic field (the yellow area in the IGW with the corresponding coherence plot showing stronger coherence in the same area). This is due to the fact that the line-minima samples the velocity field at higher layers, where the presence of magnetic fields may lead to the reflection of these waves resulting in positive phase difference.

\subsection{Dependence of phase difference on vertical travel distance}

The change in the phase spectra for different pairs of heights motivates us to look at how they vary as a function of vertical distance between the measurement heights. The dependence of the phase lag and coherence with distance between the two velocity measurements give us a sense of the height separation in the atmosphere for which the phase difference can be reliably measured. To examine this further, we choose a layer as a reference layer and compute $k_{\mathrm{h}}-\omega$ phase difference spectra for different atmospheric layers relative to this reference layer. We can then pick a representative $k_{\mathrm{h}}$ and $\omega$ that fall within the region where the bulk of the IGWs emission occurs and show the phase difference as a function of the travel distance between the reference layer and any height in the atmosphere.

In Fig. 10 we show the phase difference as a function of travel distance relative to the reference height of $z=0 \mathrm{~km}$ (thick solid curves) for the non-magnetic model (blue: Sun-v0) and the magnetic model (red: Sun-v100). The phase difference shown here is computed with the velocity field obtained directly from the simulation. The $90 \%$ confidence bounds for the phase difference estimates, according to Sect. 4.2, are represented by the shaded area of the corresponding colour. Also shown as thin solid curves are the phase differences relative to a selection of other reference layers, $z=400,600,800 \mathrm{~km}$, along with the corresponding $90 \%$ confidence bounds. The dependence of the phase propagation on the magnetic field can be clearly seen in the sign of the phase difference. Both the non-magnetic and magnetic models show negative phase difference up to around $300 \mathrm{~km}$, which means that the waves are propagating in the upward direction. Beyond the vertical travel distance of $400 \mathrm{~km}$ for the $z=0 \mathrm{~km}$ reference layer, the phase measurement become unreliable for the magnetic case. For the non-magnetic model, the phase difference can be estimated even beyond a vertical 

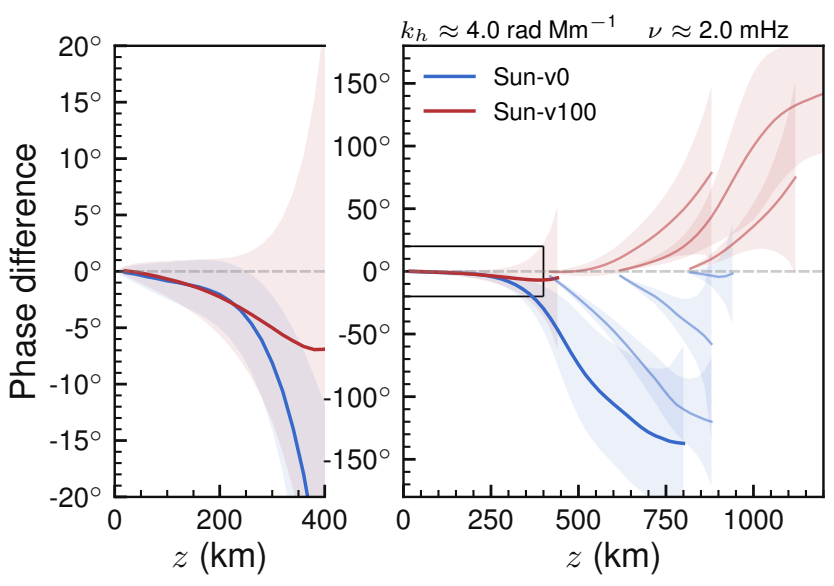

Fig. 10. Phase difference between the reference layer, $z=0$, and layers of constant geometrical scale for a given $k_{\mathrm{h}}$ and $\omega$ (thick solid red and blue curves). The left plot is a rescaled part of the small region in the right plot. The thin solid lines show three other reference layers $(z=400,600,800 \mathrm{~km})$. The $90 \%$ confidence bounds for the phase difference estimate is represented by the shaded area.

travel distance of $750 \mathrm{~km}$. Comparing the phase difference with respect to the other reference layers, this separation distance becomes shorter as we move up in the atmosphere. The sign of the phase difference in the case of the non-magnetic model remains negative (upward-propagating IGW), relative to any reference layer that is considered. On the other hand, for the magnetic models, the phase propagation relative to the reference height above $z=400 \mathrm{~km}$ is always positive, suggesting the downward propagation of waves in the presence of magnetic field.

\subsection{Dependence of coherence on vertical travel distance}

Despite the $v-v$ phase difference spectra suggesting signatures of IGW propagation, a definite validation is only possible by looking at the coherence spectra to help us in judging whether the measured phase differences are acceptable. The coherence spectra describe the quality and the reliability of the phase spectra, giving us a measure of the correlation of the two vertical velocity signals. Looking at Figs. 7-9, the coherence spectra tends to zero in specific regions of the $k_{\mathrm{h}}-\omega$ space, rendering any interpretation of the phase difference in that Fourier region unreliable. It is clear that the coherence depends on the height separation between the velocity measurements, mainly because the waves have to travel a long distance and they tend to loose their coherency with distance. Another reason could be that the surfaces of constant optical depth on which the velocities are measured are very irregular relative to constant phase surfaces of the wave. Evidence in favour of this hypothesis can be seen when comparing the coherence spectra of Fe I $\lambda 5434 \AA$ (Fig. 7) and Fe I $\lambda 5576 \AA$ (Fig. 8) which show a strong coherence for the temperature insensitive Fe I $\lambda 5576 \AA$ line.

An additional factor that may influence the coherence is the angle of propagation with respect to the vertical for a wave of a given frequency, which is strictly governed by the local Brunt-Väisälä frequency $(N)$. A wave launched at a specific frequency, in the absence of any non-linear interaction will eventually follow a curved trajectory if the local $N$ changes with height. Therefore, the coherency of the waves propagating between two heights for a given Fourier frequency may locally change over the field of view. Although the $N$ in our model atmospheres

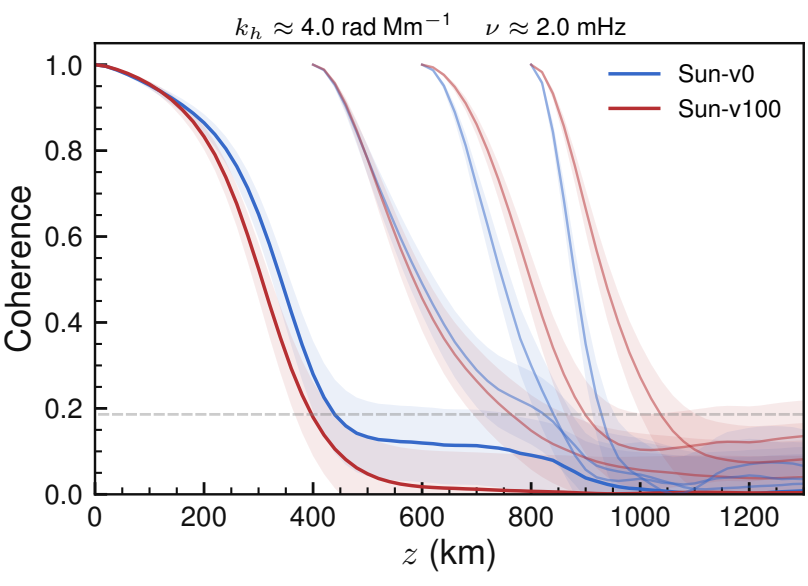

Fig. 11. Coherence between the $z=0$ layer and layers of constant geometrical height for a given $k_{\mathrm{h}}$ and $\omega$. The zero-coherence threshold at a significance level of 0.05 is marked by the dashed line. The $90 \%$ confidence bounds for the coherence are represented by the shaded area.

change with height as described in Paper I, we note that it does not differ between the models of different average field strength, and therefore the difference in phase trajectory between waves in the models below $\beta=1$ should be negligible. Only in the higher layers does the effect of magnetic field appear to be strong. Questions that remain refer to how the magnetic field affects the coherence, or whether or not the coherence changes if magnetic fields are present.

Similar to the phase difference study carried out in the previous section, we now look at the coherence as a function of the vertical travel distance. Figure 11 shows the coherence between a selection of reference heights $(z=0,400,600,800 \mathrm{~km})$ and the layers above. Also shown are the $90 \%$ confidence bounds for the coherence estimate as background shading. The zero-coherence threshold as described in Sect. 4.1 for the specific $k_{\mathrm{h}}$ is marked by the dashed grey line. Here again we look at $k_{\mathrm{h}}=4 \mathrm{rad} \mathrm{Mm}^{-1}$ and $v=2 \mathrm{mHz}$, which falls well within the IGW excitation region. We see that the coherency drops slowly for the lower layers but sharply for the layers above. The height difference corresponds to around $400 \mathrm{~km}$, which is above the significance level, for all the models when looking at the coherency between $z=0 \mathrm{~km}$ and layers above. However, higher up, the difference corresponds around $100 \mathrm{~km}$ for the non-magnetic model and $200 \mathrm{~km}$ for our strongest magnetic model. This analysis shows us that when computing the phase spectra, it is better to choose spectral lines that form very close to each other in the atmosphere; especially in the case of higher layers.

\subsection{Energy flux}

A first-order approximation to the flux of the total energy density associated with IGWs can be directly determined from the simulation. This 'linear' wave energy flux is given by the perturbed pressure-velocity $(\Delta p-v)$ co-spectrum (Eq. (7) of Paper I) in the IGW region. In Fig. 12a, we show the vertical flux of the active mechanical energy density for the non-magnetic (Sun-v0) and magnetic (Sun-v100) models at a height of $z=560 \mathrm{~km}$. The main difference between the two is the presence of a downward flux (green-blue) in the magnetic model within the IGW branch. We obtain a total positive flux of $\sim 830 \mathrm{~W} \mathrm{~m}^{-2}$ for the Sun-v0 and $\sim 1475 \mathrm{~W} \mathrm{~m}^{-2}$ for the Sun-v100 model in the IGW region for $k_{\mathrm{h}}<10 \mathrm{rad} \mathrm{Mm}^{-1}$. The energy flux for a layer of constant optical depth, as shown in Fig. $12 \mathrm{~b}$ for $\tau_{\mathrm{R}}=1 \times 10^{-4}$, gives $\sim 320 \mathrm{~W} \mathrm{~m}^{-2}$ 
(a)
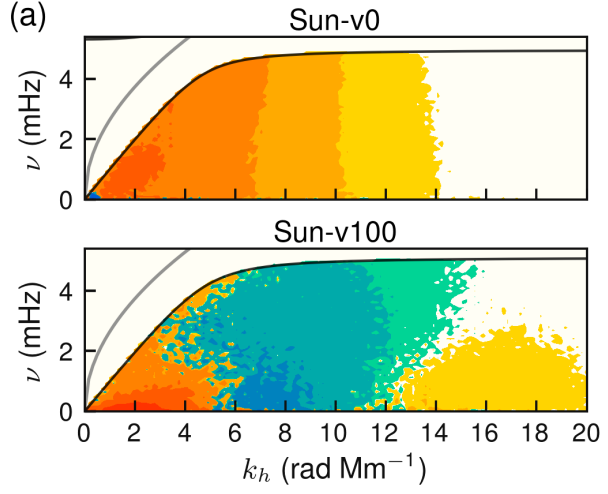

(c)
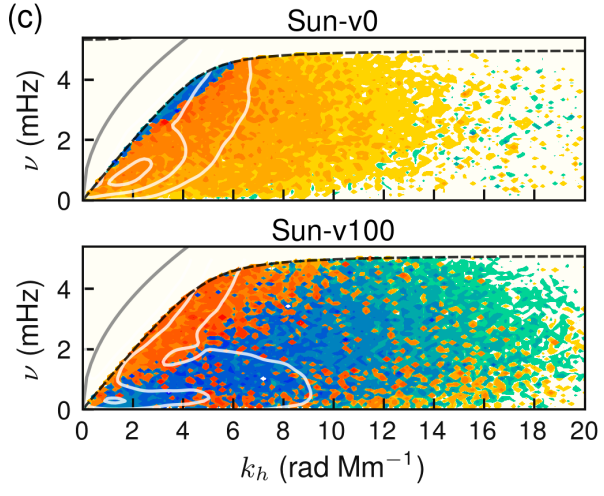
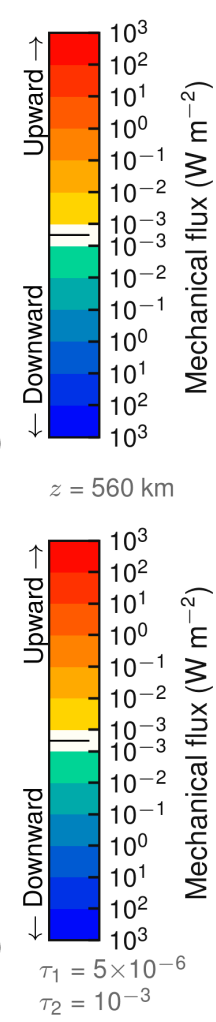

(b)
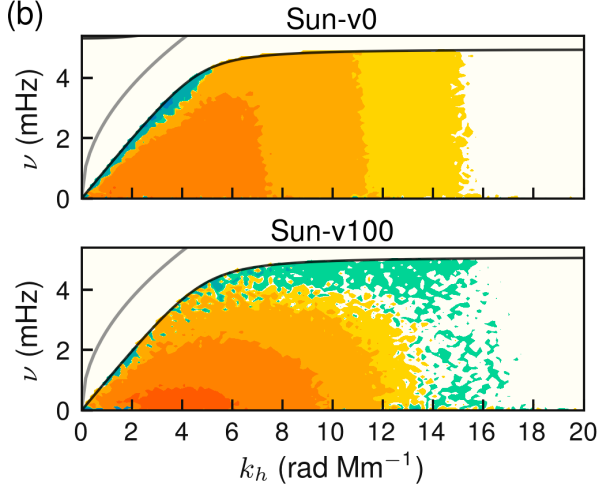

(d) Sun-v0
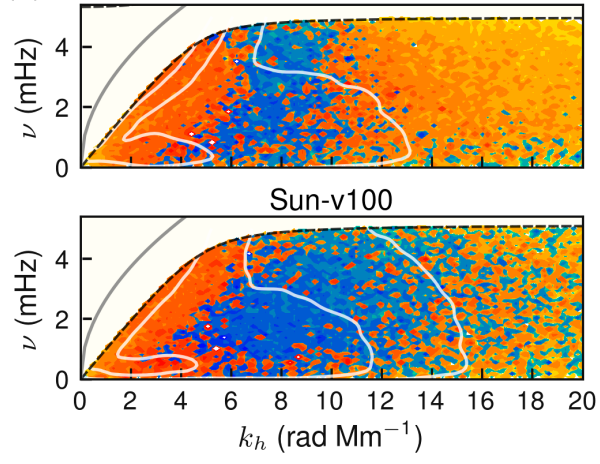

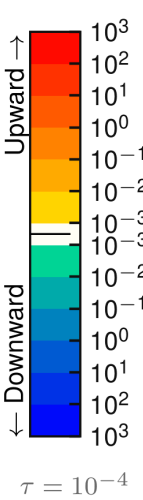

$\widehat{\sim}$

$\varepsilon$

3

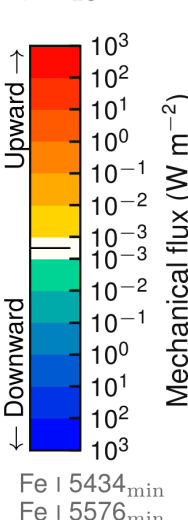

Fig. 12. Vertical mechanical flux spectra at $(a)$ a height of $z=560 \mathrm{~km}$ and at $(b)$ the Rosseland mean optical depth layer of $\tau_{\mathrm{R}}=1 \times 10^{-4}$. Also shown are the vertical energy flux spectra estimated from phase difference measurements of $(c)$ velocities at two layers of constant optical depth: $\tau_{\mathrm{R}}=5 \times 10^{-6}$ and $\tau_{\mathrm{R}}=1 \times 10^{-3}$ and from $(d)$ the line-minima shifts of the Fe I $\lambda 5434 \AA$ and Fe $\mathrm{I} \lambda 5576 \AA$ lines. The plot is masked to show only the IGW region. The grey curve marks the dispersion relation of the surface gravity waves. The black dashed curves mark the propagation boundary of acoustic-gravity waves corresponding to approximately the middle region of the two layers.

for the Sun-v0 and $\sim 617 \mathrm{~W} \mathrm{~m}^{-2}$ for the Sun-v100 model. The main discrepancy between the two flux estimates is due to the fact that the pressure perturbations are weaker in layers of constant optical depth compared to the fluctuations of pressure at a given geometrical height.

We can now compare this with the estimates obtained using phase-difference spectra, where the energy flux for IGWs is determined from the mean energy density and the group velocity, as described in Sect. 4.3. In Fig. 12c, we show the energy flux as determined from the phase difference between two layers of constant optical depth, $\tau_{1}=5 \times 10^{-6}$ and $\tau_{2}=1 \times 10^{-3}$ for the non-magnetic (Sun-v0) and magnetic (Sun-v100) models. These velocities are directly obtained from the simulation box. The difference in the $\tau_{\mathrm{R}}$ level corresponds to a height difference of $\Delta z=280-290 \mathrm{~km}$ for the non-magnetic and the magnetic models. The white contours mark selected coherence values, namely $0.2,0.4$, and 0.6. Comparing the non-magnetic and the magnetic models, we do see the change in the direction of energy transport, corresponding to upward in the non-magnetic case and a mixture of upward and downward in the magnetic case. We also note that the location of strong $v-v$ coherence in the $k_{\mathrm{h}}-\omega$ plane is different in both cases, as marked by the profile of the coherence contours. However, comparing this with the flux spectra shown in Figs. 12a and b, we see significant differences, particularly in the case of the magnetic model. The total positive flux that we obtain with the phase difference between two layers of constant optical is $\sim 1200 \mathrm{~W} \mathrm{~m}^{-2}$ and $\sim 8400 \mathrm{~W} \mathrm{~m}^{-2}$ for Sun-v0 and Sun-v10, respectively. The magnetic model shows a significant difference in the total flux between the phase-difference analysis and direct estimation.
Finally, Fig. 12d shows the energy flux as determined from the line core Doppler velocities of Fe I $\lambda 5434 \AA$ and Fe I $\lambda 5576 \AA$. The propagation boundaries in both the plots correspond to a height of $z \approx 550 \mathrm{~km}$. The mean density and the mean Brunt-Väisälä frequency are taken from this layer for the estimation of the energy flux. A height separation $(\Delta z)$ of $310 \mathrm{~km}$ for the non-magnetic model and $280 \mathrm{~km}$ for the magnetic model is assumed. The total positive flux we obtain for the IGW using the spectral line is an order of magnitude more than what we estimate from simulations using the same method. For the Sun-v0 model we obtain a total positive flux of $\sim 38200 \mathrm{~W} \mathrm{~m}^{-2}$ and for the Sun-v100 model we get $\sim 90900 \mathrm{~W} \mathrm{~m}^{-2}$. The velocities from the simulation at layers of constant optical depth are comparable to the Doppler velocities that we derive from the spectral line. However, we see that the phase differences in the simulation are significantly different from the phase differences obtained using the spectral lines. They are considerably smaller, with a value often close to zero, leading to very high phase speed and therefore very high group speed estimates. Energy flux estimates based on this method may therefore be significantly overestimated.

To summarise, we note that the energy flux from the simulation and that obtained using the spectral lines for the nonmagnetic model are significantly different in terms of both the region where the strongest flux lies and its magnitude. The magnetic models show a larger difference compared to the nonmagnetic model. The actual reason for the differences in the energy flux spectra measured with the different methods is still unclear. However, we would like to point out that relying solely on the energy estimates from the phase difference measurement 
may lead to unrepresentative results. In a realistic atmosphere, surfaces of constant optical depth show large variations. In addition to this, the velocity fields are required at two separate layers in order to determine the energy flux. The velocity field is likely to be smeared out resulting in inaccurate determination of the phase difference, and consequently the energy flux.

\subsection{Comparing wave spectra from simulations with synthetic observations}

In Paper I and Paper II, where we studied the wave spectra directly in the simulation domain, we clearly see the influence of the magnetic field on the propagation of IGWs with height. However, as this work shows, when it comes to synthetic observation, the possible signatures of the magnetic fields on the IGW propagation are not apparent. Although we clearly see signatures of IGWs in the synthetic Doppler velocities, it is hard to discern the effect of magnetic fields on the propagation of these waves unless velocity measurements from very near-lying layers are used.

The observational detection of IGWs are further challenged by the problem of coherence. In the models considered here, a reliable IGW spectrum can be measured in the near surface layers with a height difference of up to $400 \mathrm{~km}$, while in the chromosphere this drastically reduces to less than $100 \mathrm{~km}$. Given the width of formation of the line, it is quite challenging to obtain a reliable phase difference measurement in the low-chromospheric regions. Also, the more temperature sensitive line, Fe I $5434 \AA$, shows stronger incoherence than the Fe I $5576 \AA$ line.

The differences between the simulation and the synthetic observations are mostly attributable to the fact that the waves are probed at different layers. While the geometrical height is unchanged by the passage of the waves, the optical depth scale varies and may be modified by the waves, making the coherence weak and the corresponding phase difference analysis difficult. Additionally, in the geometrical height scale we have measurements from precise heights, whereas in the synthetic observation case our velocity measurements are taken over a broader region and are often smeared out. This also adversely affects the estimation of the energy flux from spectral lines.

\subsection{Comparing wave spectra from synthetic with real observations}

The measurements from the synthetic Dopplergram facilitates a direct comparison to observations. Our results are consistent with the observations by Straus et al. (2008) and Nagashima et al. (2014) in terms of the qualitative properties of the phase-difference. In order to directly compare our results with observations, we compare our results with those of Kneer \& Bello González (2011). Kneer \& Bello González (2011) looked at the Fe I $\lambda \lambda 5576 \AA$ and $5434 \AA$ lines, which were scanned quasi-simultaneously. These latter authors estimated the power, phase, and coherence spectra from the line minimum velocities for the two lines. They report a minimum phase difference of approximately $-40^{\circ}$ for $k_{\mathrm{h}} \approx 4 \mathrm{rad} \mathrm{Mm}^{-1}$ and $v \approx 2.5 \mathrm{mHz}$, which is consistent with our results as evident from Fig. 9 b. The authors report that for $k_{\mathrm{h}} \geq 13 \mathrm{rad} \mathrm{Mm}^{-1}$ the phases become random, which is also seen in our synthetic data (not shown in Fig. 9b). In terms of coherence, they obtain a maximum of 0.85 in the IGW regime, which is slightly higher than the coherence we see in our simulated models and the spectral lines. Our study provides an indication that the simulations reproduce the observations and confirm the existence of IGWs.

\subsection{Future observational strategy}

Our study of the two neutral Fe lines shows that for the analysis of lines with a high-excitation potential, magnetically non-sensitive lines may be better for studying IGWs. Some lines possibly well-suited for studying the IGWs, apart from the two lines studied here, are Ni I 4912, Fe I 5691, Fe I 7090, and Fe II 722.45 (Cabrera Solana et al. 2005). We suggest future observations to look at either a single weak line and measure the phase and coherence from the different Doppler velocities, or to look at two spectral lines that are in approximately the same region in the atmosphere. Ongoing work with predominantly horizontal fields shows upward propagation for IGWs (downward phases) even in low plasma- $\beta$, indicating that these waves may still be important for chromospheric layers. We infer from our analysis that the photospheric detection of IGWs is relatively straightforward, but observing waves in the higher layers might be hindered by the availability of chromospheric lines that have a narrow line-of-formation profile. Progress may come from exploring other techniques that provide more localised measurement of the solar velocity field, like relying on a combination of multiple lines (Wunnenberg et al. 2002). Furthermore, any analysis should certainly consider NLTE as evident from the difference in the $R F_{\mathrm{v}}$ that we estimate using LTE.

\section{Conclusions}

Here, we present a study of internal gravity waves detected in synthetic observations that cover a range of quiet solar magneto-atmospheric conditions. A selection of models of varying average magnetic flux density were simulated with the state-of-the-art radiative-MHD code $\mathrm{CO}^{5} \mathrm{BOLD}$. Using these time-evolving 3D dynamic models as input, we synthesised spectral lines at individual snapshots to look at signatures of waves in the intensity and derived Doppler velocity maps. We compared the wave spectra in the simulated physical volume with that obtained from the synthetic observations. In this study, we relied on the different estimates of the Doppler velocities to infer the 2D velocity field at various layers in the solar atmosphere in order to carry out the phase and coherence analyses. One of our main aims was to investigate the feasibility of detecting signatures of IGWs in the emergent wave spectra. Furthermore, we were motivated to study the influence of magnetic field on the waves and its manifestation in the diagnostic spectra. In addition to gaining an understanding of how to detect IGWs in real observations, we also aimed to examine the method for observational estimation of the energy flux of these waves, an aspect which is important in the context of their contribution to the total wave energy budget of the solar atmosphere.

The time series of the Doppler velocities estimated from two non-magnetic neutral iron lines Fe I $\lambda \lambda 5434 \AA$ and $5576 \AA$ were used for the study of waves. We carried out Fourier analyses to derive the power, phase, and coherence spectra in the $k_{\mathrm{h}}-\omega$ dispersion relation diagram to separate IGWs from acoustic waves. The characteristic properties of the phases in the dispersion relation diagram clearly show us that the waves are indeed observable in velocity maps obtained from observations. However, the coherency varies strongly within different regions of the IGW domain rendering any phase analysis in those regions unreliable. Furthermore, any detection of the effect of magnetic field on these waves is complicated by the properties of the selected lines and their formation height. Our analysis shows that unlike the clear signature of IGW propagation seen in simulations, the observation signatures do not allow for a straightforward 
interpretation on the effect of magnetic field on the propagation of these waves. By comparing energy flux directly obtained from the simulation with that determined from the spectral lines, we come to the conclusion that the specific choice of spectral lines plays an important role; the types of lines needed for such an analysis tend to smear out the velocity field depending on the width of the line-formation region and their spatial separation, resulting in significantly smaller phase differences which can lead to overestimation of the energy flux of IGWs by an order of magnitude. Our understanding is that lines that are temperature insensitive and non-magnetic with narrow line-formation ranges may be preferable for detecting and studying internal waves in the solar atmosphere.

Acknowledgements. GV would like to thank B. Fleck, O. Steiner, J. M Borrero, N. Bello González, A. Pastor Yabar, and J. Bruls for the helpful discussions. We thank the referee for his detailed comments and suggestions that significantly improved the presentation of the paper. This work was supported by the Deutsche Forschungsgemeinschaft, DFG grant RO 3010/3-1.

\section{References}

Barklem, P. S., Anstee, S. D., \& O’Mara, B. J. 1998, PASA, 15, 336 Bello González, N., Flores Soriano, M., Kneer, F., \& Okunev, O. 2009, A\&A, 508, 941

Bello González, N., Flores Soriano, M., Kneer, F., Okunev, O., \& Shchukina, N. 2010, A\&A, 522, A31
Berrilli, F., Consolini, G., Pietropaolo, E., et al. 2002, A\&A, 381, 253

Borrero, J. M., Jafarzadeh, S., Schüssler, M., \& Solanki, S. K. 2017, Space Sci. Rev., 210, 275

Cabrera Solana, D., Bellot Rubio, L. R., \& del Toro Iniesta, J. C. 2005, A\&A, 439, 687

Couvidat, S., Rajaguru, S. P., Wachter, R., et al. 2012, Sol. Phys., 278, 217

Eibe, M. T., Mein, P., Roudier, T., \& Faurobert, M. 2001, A\&A, 371, 1128

Fleck, B., Couvidat, S., \& Straus, T. 2011, Sol. Phys., 271, 27

Freytag, B., Steffen, M., Ludwig, H. G., et al. 2012, J. Comput. Phys., 231, 919

Gustafsson, B., Edvardsson, B., Eriksson, K., et al. 2008, A\&A, 486, 951

Hannan, E. J. 1990, Multiple Time Series, Wiley Series in Probability and Statistics (Wiley)

Kneer, F., \& Bello González, N. 2011, A\&A, 532, A111

Kramida, A., Ralchenko, Yu., Reader, J., \& and NIST ASD Team 2018, NIST Atomic Spectra Database (ver. 5.6.1), [Online]. Available: https: //physics.nist.gov/asd [2019, September 30]. (National Institute of Standards and Technology: Gaithersburg, MD)

Nagashima, K., Löptien, B., Gizon, L., et al. 2014, Sol. Phys., 289, 3457

Newington, M. E., \& Cally, P. S. 2010, MNRAS, 402, 386

Newington, M. E., \& Cally, P. S. 2011, MNRAS, 417, 1162

Rutten, R. J., \& Krijger, J. M. 2003, A\&A, 407, 735

Scherrer, P. H., Bogart, R. S., Bush, R. I., et al. 1995, Sol. Phys., 162, 129

Socas-Navarro, H., de la Cruz Rodríguez, J., Asensio Ramos, A., Trujillo Bueno, J., \& Ruiz Cobo, B. 2015, A\&A, 577, A7

Straus, T., \& Bonaccini, D. 1997, A\&A, 324, 704

Straus, T., Fleck, B., Jefferies, S. M., et al. 2008, ApJ, 681, L125

Vigeesh, G., Jackiewicz, J., \& Steiner, O. 2017, ApJ, 835, 148

Vigeesh, G., Roth, M., Steiner, O., \& Jackiewicz, J. 2019, ApJ, 872, 166

Wunnenberg, M., Kneer, F., \& Hirzberger, J. 2002, A\&A, 395, L51 